\title{
Developing and Evaluating a Game for the Assessment of Spatial Memory Using Auditory Stimuli
}

\author{
M. Loachamín, M. C. Juan, M. Méndez, E. Pérez and M. J. Vicent
}

\begin{abstract}
The combination of natural user interfaces and smart devices has become a new technological option to be exploited for the assessment of spatial memory. In this paper, we present an interactive game for assessing spatial memory using auditory stimuli. The distinct feature of our game is that it assesses the two types of information (i.e., acoustic and spatial) at the same time. We compared the performance of 100 children and 48 adults on the game with traditional neuropsychological tests. The performance on the game correlated with the performance on other traditional neuropsychological tests for the assessment of spatial short-term memory. Children and adults considered our game as easy to use, and all of them were satisfied with it. The results suggest that our game could be a valid tool for the identification of alterations in the spatial memory in both children and adults.
\end{abstract}

Index Terms-Natural User Interfaces, Kinect, smart devices, Karotz, short-term spatial memory, auditory stimuli

\section{INTRODUCCIÓN}

$\mathrm{L}$ A CRECIENTE evolución de la tecnología ha permitido el desarrollo de nuevos sistemas y el uso de diferentes tipos de dispositivos con distintos propósitos. Dicha evolución incrementa las oportunidades para el desarrollo de herramientas y tecnologías, así como la fabricación de nuevos dispositivos inteligentes y nuevas interfaces de usuario naturales (NUI). Los dispositivos inteligentes son recursos tecnológicos capaces de comunicarse. Esta comunicación puede ser entre dispositivos similares a través de un protocolo de comunicación o con humanos a través de una interfaz de usuario. Las NUI permiten la interacción natural, en las que el propio cuerpo es el responsable de transmitir instrucciones a las aplicaciones, sin utilizar mandos o dispositivos de entrada. Las NUI utilizan dispositivos con sensores para capturar el movimiento del cuerpo, permitiendo al usuario interactuar sin necesidad de tener contacto físico con otros dispositivos de control.

Una de las habilidades cognitivas más críticas en seres humanos es almacenar el recuerdo de estímulos que se han

M. Loachamín, Instituto Universitario de Automática e Informática Industrial, Universitat Politècnica de València, Valencia, España, mrenan@dsic.upv.es

M. C. Juan, Instituto Universitario de Automática e Informática Industrial, Universitat Politècnica de València, Valencia, España, mcarmen@dsic.upv.es

M. Méndez, Departamento de Psicología y Sociología, Universidad de Zaragoza, Zaragoza, España, mmendez@unizar.es

E. Pérez, Departamento de Psicología Evolutiva y de la Educación, Universidad Autónoma de Madrid, Madrid, España, elena.perezh@uam.es

M. J. Vicent, Instituto Universitario de Automática e Informática Industrial, Universitat Politècnica de València, Valencia, España, mvicent@upv.es experimentado en el pasado. Desde una perspectiva psicológica, existe interés en investigar la capacidad de aprender información espacial y auditiva. La razón es que son procesos significativos en la vida diaria. La memoria se puede dividir en memoria a corto plazo y a largo plazo, dependiendo de si la información a memorizar es útil por un período de tiempo limitado o es relevante para ser almacenada de forma estable ante cualquier necesidad futura [1]. Las memorias espacial y auditiva tienen la capacidad de almacenar representaciones de estímulos espaciales y auditivos, respectivamente. La de tipo espacial nos permite encontrar un lugar visitado con anterioridad, seguir una ruta después de consultar un mapa o recordar el lugar dónde dejamos nuestras pertenencias, entre otros ejemplos [2]. El aprendizaje espacial se asocia con resultados académicos [3]. El aprendizaje auditivo facilita tomar notas mientras se escucha, la expresión escrita y oral [4]. Algunos problemas de aprendizaje y de comportamiento están relacionados con las deficiencias en este proceso [5]. Además, muchas experiencias de aprendizaje requieren considerar la información auditiva y espacial simultáneamente. Algunos ejemplos son la orientación espacial y la identificación de personas u objetos. Dado que no existen procedimientos para evaluar la orientación espacial con estímulos auditivos, sus implicaciones para los diferentes tipos de discapacidades siguen siendo inciertas.

Las aplicaciones o herramientas relacionadas con la memoria espacial pueden ser para evaluación o para entrenamiento. Las aplicaciones para evaluación permiten determinar el estado de la memoria de una persona en un momento determinado. Los resultados de dicha evaluación permiten la identificación temprana del desarrollo atípico en niños, o bien si un adulto presenta alteraciones en su memoria, como es el caso de enfermos de Alzheimer, otros tipos de demencia con alteraciones de la orientación, pacientes con secuelas tras sufrir un accidente cerebrovascular o traumatismo craneal, etc. Algunas de las aplicaciones existentes se centran en el déficit de memoria general, por ejemplo, Cuerda \& Gallud [6] presentaron Forgettify, que es una app para Android y se gestiona utilizando un cliente web. La app ayuda en actividades de la vida diaria (p. ej., cómo poner la lavadora) a través de video guías. El cliente web lo utilizan profesionales y familiares para personalizar los contenidos del usuario. La app se evaluó con 7 pacientes con algún tipo de demencia en una fase leve de la enfermedad y 4 profesionales. La tarea principal del estudio consistía en seguir un video guía que explicaba cómo realizar un avión de papel. El participante tenía que hacer 
el avión simultáneamente. El estudio analizó si los participantes necesitaban asistencia adicional y la satisfacción con la app. Los participantes con menos conocimientos de informática necesitaron más asistencia. La satisfacción fue mayor en los participantes más jóvenes.

Por lo que sabemos, no se ha explotado la combinación de NUI y dispositivos inteligentes para evaluar procesos cognitivos, y en especial, para evaluar la memoria espacial utilizando estímulos auditivos de forma inalámbrica y atractiva. El desarrollo de nuevos sistemas para la evaluación neuropsicológica representa una herramienta importante para la evaluación de la memoria como una función cognitiva que está vinculada a diversas actividades intelectuales y sociales, y también, como se ha comentado, para la identificación de alteraciones en la misma. Herramientas de este tipo podrían contribuir a una mejor comprensión de la influencia de diferentes variables en la función cognitiva.

En este artículo presentamos el desarrollo del primer juego para evaluar la memoria espacial a corto plazo utilizando estímulos auditivos, que incorpora NUI y dispositivos inteligentes. Para identificar las ventajas de nuestro juego respecto a tests tradicionales se ha llevado a cabo un estudio. El objetivo de dicho estudio es demostrar el potencial del juego para evaluar la memoria espacial a corto plazo. Para ello, se comparan los resultados obtenidos usando nuestro juego con la aplicación de test tradicionales aplicados a niños y adultos. La hipótesis principal es que los resultados del juego reflejarán la capacidad del mismo para evaluar la memoria espacial a corto plazo tanto para niños como para adultos, ofreciendo correlaciones significativas respecto a procedimientos tradicionales.

\section{ESTADO DEL ARTE}

\section{A. Interfaces de usuario naturales}

La aparición de sensores de profundidad (p. ej., Kinect o Leap Motion) ha permitido que se puedan usar como dispositivos de entrada para interfaces de usuario naturales. Dichos sensores se han utilizado con distintos objetivos y para una gran variedad de aplicaciones, como por ejemplo aprendizaje [7]; rehabilitación [8] reconocimiento del lenguaje de signos [9] [10]; o diagnóstico de parálisis facial [11]. La Tabla I muestra las características de los sensores de profundidad comerciales más utilizados, según Cheng et al. [9].

Como se observa en la Tabla I, Kinect, en sus dos versiones, se puede utilizar para identificar partes del cuerpo y Leap Motion se centra en los gestos de la mano. Leap Motion se ha utilizado, por ejemplo, para facilitar la comunicación de personas con trastornos de comunicación verbal [12]. En nuestro caso, y dado que se pretendía identificar que los usuarios levantasen los brazos se utilizó Kinect. Para ampliar la información sobre sensores de profundidad, se recomienda consultar el artículo publicado por Cheng et al. [9].

\section{B. Dispositivos inteligentes}

Los dispositivos inteligentes, especialmente aquellos con apariencia humana (robots), han contribuido al desarrollo de varias aplicaciones. Uno de los robots más utilizados hasta la fecha, ha sido el robot NAO, que se ha utilizado, por ejemplo,
TABLA I

SENSORES DE PROFUNDIDAD COMERCIALES

\begin{tabular}{lllll}
\hline \hline \multicolumn{1}{c}{ Sensor } & Resolución & Rango & Precisión & $\begin{array}{c}\text { Puntos de } \\
\text { unión }\end{array}$ \\
\hline Kinect 1.0 & $320 \times 240$ & $0.8-4.0 \mathrm{~m}$ & $4.00 \mathrm{~mm}$ & 20 cuerpo \\
Leap Motion & $640 \times 240$ & $25-600 \mathrm{~mm}$ & $0.01 \mathrm{~mm}$ & 27 mano \\
Kinect 2.0 & $512 \times 484$ & $0.8-4.5 \mathrm{~m}$ & $1.00 \mathrm{~mm}$ & 25 cuerpo \\
\hline \hline
\end{tabular}

con niños autistas para promover la comunicación y habilidades sociales como parte de un programa de rehabilitación [13]; o en KindSAR para ayudar a niños preescolares en su desarrollo cognitivo a través de la interacción social [14].

También se han utilizado otros robots, por ejemplo, Robovie, que es un robot humanoide autónomo $(1.2 \mathrm{~m}$ de alto, $50 \mathrm{~cm}$ de diámetro, y $40 \mathrm{~kg}$ de peso) con ojos y manos de aspecto humano. Moriguchi et al. [15] utilizaron Robovie para que niños de entre 4 y 6 años pudieran aprender palabras. Se han empleado robots para desarrollar el pensamiento computacional, por ejemplo, para estudiantes de secundaria en el que se utilizó un robot construido mediante Lego Mindstorm NTX [16]. Enríquez et al. [16] obtuvieron resultados satisfactorios en cuanto a utilidad e interés. Los resultados de aprendizaje relacionados con implementación y construcción de algoritmos también fueron positivos. Por lo que sabemos, el único estudio que ha utilizado hasta la fecha el robot Karotz es el presentado por de Graaf et al. [17]. Karotz es el robot incluido en nuestro sistema. De Graaf et al. [17] instalaron Karotz en la casa de personas mayores. El objetivo era mejorar su salud. La función del robot era la de un 'asistente personal' que se interesaba por sus progresos y les hacía recomendaciones, como por ejemplo controlar su peso.

\section{Evaluación de la memoria espacial}

La evaluación de la memoria espacial utilizando estímulos visuales se ha llevado a cabo utilizando distintos tipos de aplicaciones. Por ejemplo, ARSM Task [18] es un sistema de realidad aumentada para evaluar la memoria espacial en niños, utilizando para ello una Tablet protegida con una carcasa. La sala en la que se realizó el estudio era de $5 \mathrm{~m}^{2}$. En ella se colocaban unas cajas reales y en su interior se encontraban los objetos virtuales a localizar. El sistema constaba de 7 niveles, en los que el número de cajas y objetos a recordar se incrementaba. En el Nivel I había 2 cajas y 1 objeto a recordar, en el Nivel II, cuatro cajas y 2 objetos a recordar, etc. El participante tenía que recorrer la sala para ver y recordar la ubicación de los objetos y después para identificar dichas ubicaciones. Juan et al. [18] encontraron similitudes en los resultados utilizando ARSM Task y métodos tradicionales. Recientemente, se ha presentado un segundo sistema de realidad aumentada con el mismo objetivo, pero basado en SLAM (Simultaneous Localization and Mapping) [19] [20]. Este sistema también se centra en estímulos visuales, pero permite la colocación de objetos virtuales en un entorno real sin añadir elementos adicionales a la escena. Munoz et al. [19] demostraron que recorrer el entorno y ver los objetos virtuales en el mundo real ayuda a los participantes a recordar la ubicación de los objetos virtuales en el entorno real si se compara con ver fotografías del entorno aumentado. Nuestra propuesta comparte la misma idea de que los estímulos a utilizar deben estar ubicados en el entorno real [19] [20]. En 
MnemoCity Task [21], se utilizó un entorno de realidad virtual, con estereoscopía pasiva e interacción natural para evaluar la memoria espacial en niños. En el estudio se compararon dos tipos de interacción: un gamepad; y un volante (con un control Wii Remote ${ }^{\mathrm{TM}}$ en su interior) para determinar los giros del usuario y Wii Balance Board ${ }^{\mathrm{TM}}$ para determinar los pasos del participante (velocidad). Se diseñó un entorno que recreaba un parque en el que se colocaron unas mesas sobre las que aparecían los elementos que el usuario debía localizar. Rodríguez et al. [21] también obtuvieron correlaciones entre su sistema y métodos tradicionales. Cárdenas et al. [22] desarrollaron un laberinto virtual para evaluar la memoria espacial en adultos. En dicho sistema, el participante tenía que recordar el recorrido en el laberinto para encontrar la salida. En su estudio se compararon dos tipos de interacción: un gamepad y una bicicleta (el pedaleo indicaba la velocidad y el giro del manillar condicionaba el giro del avatar presente en el mundo virtual). Los resultados de los participantes obtenidos con el laberinto correlacionaron con métodos tradicionales. Sin embargo, por lo que sabemos, no existen estudios que hayan evaluado la memoria espacial utilizando estímulos auditivos.

\section{DESARROLLO}

\section{A. Diseño del juego}

El proceso de diseño del juego se centró en el usuario. El juego debía contemplar su uso por diferentes colectivos: niños, adultos e invidentes. Se llevaron a cabo varias sesiones de diseño participativo con usuarios potenciales (niños y adultos), expertas psicólogas y expertos en informática gráfica e interacción persona-ordenador. En primer lugar, se estudió el tipo de ambiente más adecuado (con o sin pistas visuales), la distribución de las fuentes de sonido, el tipo de interacción y la dinámica del juego. Para la distribución de las fuentes de sonido, tras varias propuestas se consideró que dichas fuentes tenían que estar frente al usuario, no detrás de él, es decir, el sonido debía llegar al usuario de frente. Por lo tanto, máximo se podría considerar un ángulo de $120^{\circ}$. Con este ángulo se estimó el número de fuentes de sonido que se podrían llegar a utilizar y se determinó que el número ideal era 5. Para el tipo de interacción fue vital la contribución de los usuarios potenciales. Una vez identificada la interacción natural como el tipo de interacción a utilizar, se determinó qué tipo de gestos eran más fáciles para los usuarios. Para ello se realizó un estudio preliminar con quince niños. Se identificó que el gesto más apropiado era levantar ambos brazos al mismo tiempo delante de la fuente de sonido. A continuación, se estudiaron las alternativas en cuanto a los dispositivos a utilizar para la emisión de los sonidos. Una propuesta básica era el uso de altavoces distribuidos por la sala para la emisión de sonidos y controlados desde un ordenador central. Sin embargo, altavoces distribuidos por la sala resultaban poco atractivos. Como alternativa se identificó la posibilidad de utilizar Karotz. Era un dispositivo con apariencia atractiva, que podía emitir sonidos y luces, mover las orejas y un botón de interacción en la parte superior. Por todo ello, fue el dispositivo seleccionado. También se plantearon los temas a utilizar en el juego y cuáles serían los que se utilizarían en cada nivel. El número de sonidos-fuentes a memorizar para cada nivel se estableció en orden de dificultad creciente, de 1 a 5 , basándonos en la capacidad humana para memorizar sonidos (un máximo de 9 elementos en el caso de adultos [23]). El diseño del juego se definió como resultado de estas sesiones.

\section{B. Descripción general del juego}

El juego permite comprobar la capacidad del usuario para detectar y localizar estímulos auditivos que se emiten en diferentes posiciones de un área de juego (Fig. 1). Para la emisión de sonidos se ha utilizado un dispositivo inteligente (robot) con la forma de conejo, Karotz. El juego utiliza una interfaz natural de usuario mediante gestos, utilizando el sensor Kinect de Microsoft. Concretamente, Kinect se utiliza para detectar que los usuarios levantan los brazos frente a un determinado Karotz con el fin de seleccionarlo. El usuario debe memorizar las posiciones de los Karotz. Los Karotz no tienen ninguna característica física que los distinga unos de otros, solo su colocación en la sala. Los Karotz pueden mover las orejas y encender la luz que se encuentra en el centro del dispositivo. El área de juego fue de $5 \mathrm{~m}^{2}$. Esta área se delimitó en una habitación con dimensiones mayores. Las paredes estaban cubiertas con papel de embalaje para eliminar cualquier pista visual. Se colocó césped artificial en el suelo para guiar las rutas, y se utilizó una alfombra para indicar la posición inicial del usuario. La altura de los Karotz se ajustó a la altura de los usuarios colocando cajas de cartón sobre las mesas y encima de dichas cajas se colocaban los Karotz. Concretamente, los cinco Karotz se disponen en la sala con la siguiente configuración: Karotz $1\left(-60^{\circ}\right)$; Karotz $2\left(-30^{\circ}\right)$; Karotz $3\left(0^{\circ}\right)$; Karotz $4\left(+30^{\circ}\right)$; Karotz $5\left(+60^{\circ}\right)$.

El juego incluye un total de 45 estímulos auditivos, que se emiten aleatoriamente en diferentes posiciones para evitar repeticiones o secuencias preestablecidas. El juego se compone de cinco niveles diferentes (Fig. 2). Cada nivel consta de 3 ensayos o intentos y está relacionado con un tema de sonidos específico, que son los siguientes: naturaleza, fiesta, granja, casa y una gran ciudad. La diferencia entre niveles radica en el número de sonidos a utilizar por ensayo, que irá incrementándose en 1 en cada nivel posterior. Por ejemplo, Nivel I (1 estímulo por ensayo); Nivel II (2 estímulos por ensayo); o Nivel V (5 estímulos por ensayo).

Cada nivel cuenta con dos fases: la fase de búsqueda y la fase de localización. En la fase de búsqueda, el usuario aprende los sonidos y su ubicación. Primero, el usuario escucha las instrucciones a través de los altavoces. Luego, escucha el sonido que emite un Karotz constantemente y memoriza su ubicación. Mientras se reproduce el sonido, el participante se mueve para situarse frente al Karotz que emite el sonido y levanta los brazos para su selección. El Karotz emite un mensaje para indicar que ha entendido el gesto. A los usuarios se les indica que tienen que escuchar el mensaje de los dispositivos tras levantar los brazos. Si el dispositivo no emite dicho mensaje, el usuario debe repetir la acción.

En la fase de localización se evalúa la memoria espacial del participante. Primero, el usuario escucha las instrucciones a través de los altavoces y también el sonido que tiene que localizar. En esta fase de localización, el usuario tiene que recordar la ubicación del Karotz que emitió el estímulo (sonido). Los estímulos sólo se emiten una vez. El usuario tiene 


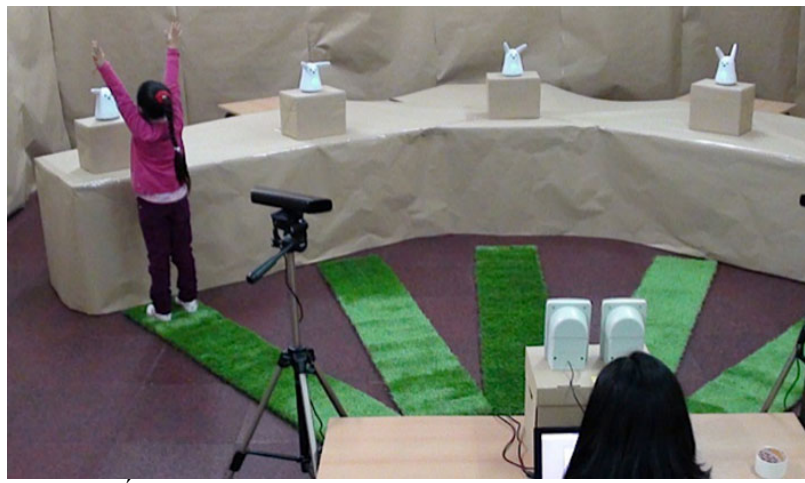

Fig. 1. Área de juego.

que moverse a la localización correcta y levantar los brazos delante del Karotz para seleccionarlo. Después, debe volver a la posición inicial. El juego almacena todas las respuestas (aciertos o fallos).

Un ensayo se supera con éxito si todos los sonidos del mismo se localizan correctamente. Si no se localiza correctamente un sonido, el ensayo no ha sido superado. Si hay, al menos, un ensayo con éxito de los 3 ensayos de un nivel dado, el usuario ha superado dicho nivel y avanza al siguiente nivel. El participante debe realizar los 3 ensayos de un nivel, independientemente de que haya acertado todos los estímulos del primer ensayo. Si falla en los 3 ensayos en cualquiera de los niveles, el juego termina. El juego también termina cuando el participante completa el nivel V.

\section{Hardware y Software}

Se utilizan dos sensores Kinect de Microsoft 1.0 para abarcar todo el área de juego y cinco Karotz. Los sensores Kinect de Microsoft 1.0 incluyen una cámara RGB con una resolución de $640 \times 480$ píxeles, una cámara infrarroja y un proyector de infrarrojos. Los Karotz tienen forma de conejo y miden $30 \mathrm{~cm}$ de altura. Pueden conectarse a Internet. Tienen altavoces, una cámara web, un LED de luz (en su parte central), y pueden mover sus orejas. Las luces pueden ser de diferentes colores. En nuestro juego, los códigos de colores son los siguientes. El color verde indica que el juego está listo para comenzar. El color azul indica éxito. El color naranja indica fallo. El color blanco indica que el Karotz está esperando la respuesta del usuario. El color rojo indica que no se ha comunicado con el servidor.

Se utilizó un ordenador con controlador de puertos USB separados para poder utilizar dos sensores Kinect simultáneamente. Además, este ordenador se utilizó como servidor. Se usaron dos altavoces convencionales para dar instrucciones durante el juego.

Se utilizó Visual Basic 2008 Express Edition para el desarrollo del sistema que gestiona el juego y la interfaz gráfica para el supervisor. Para programar los sensores Kinect, se utilizó Visual C ++ 2010 Express Edition, Kinect SDK 1.8, OpenNI 2.0 SDK y Nite. El sistema tiene tres módulos: (a) un módulo para configurar y administrar los Karotz, sus IPs y el IP del servidor de sonidos; (b) un módulo para gestionar la comunicación entre los Karotz y los Kinect; (c) un módulo para registrar la información del participante y para el juego, propiamente dicho (proceso de evaluación).

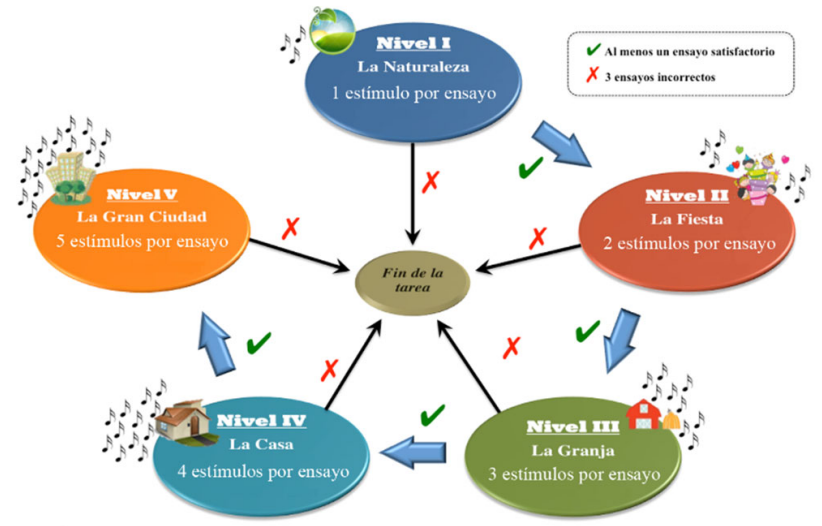

Fig. 2. Niveles del juego.

El sistema tiene una interfaz gráfica que permite al supervisor introducir el código del participante, su fecha de nacimiento y género. La función del supervisor es supervisar el progreso del participante observando dicha interfaz, es decir, nivel, ensayos, éxitos y fallos. El supervisor no controla a los Karotz.

\section{ESTUDIO}

\section{A. Muestra del estudio}

El estudio consta de una muestra de 148 participantes. De los cuales, 100 fueron niños de edades comprendidas entre 5 y 10 años, y 48 adultos de edades comprendidas entre 18 y 28 años. La edad media de los niños fue de $7.49 \pm 1.69$ años, y de los adultos $20.98 \pm 2.76$. La distribución de la muestra de los niños fue de 50 niños $(50 \%)$ y de 50 niñas $(50 \%)$. La distribución de la muestra de los adultos fue de 24 hombres $(50 \%)$ y de 24 mujeres $(50 \%)$. El total de participantes se dividió en tres grupos: Grupo A (niños de 5 a 7 años), Grupo B (niños de 8 a 10 años), y Grupo C (adultos de 18 a 28 años).

El estudio se llevó a cabo de conformidad con la Directiva Europea 2001/20/EC y la Declaración de Helsinki para la investigación biomédica con seres humanos. Los adultos recibieron información por escrito sobre los objetivos y procedimiento del estudio y firmaron consentimiento informado para participar en el mismo. De igual modo, los padres de los niños recibieron la misma información y firmaron consentimiento informado para que sus hijos participaran en el estudio. A todos los niños se les pidió su consentimiento verbal. Los participantes tuvieron plena libertad para abandonar el estudio en cualquier momento. El estudio fue aprobado por el Comité de Ética de la Universitat Politècnica de València y de la Universidad Autónoma de Madrid.

\section{B. Variables utilizadas en el análisis}

Se utilizan tres variables relacionadas con el juego: Número de Niveles Correctos (NNC), Número de Estímulos Auditivos Acertados (NEA) y Tiempo Total (TT). La variable NNC es el número de niveles correctamente superados, $\mathrm{y}$ mide la capacidad para recordar los estímulos auditivos y sus ubicaciones. La variable NNC puede tener un valor de cero a cinco, en función de los niveles superados con éxito. NEA es el número de estímulos auditivos que el participante pudo 
localizar con éxito. TT es el tiempo total en minutos que el participante empleó para completar el juego.

También se evaluó la capacidad para recordar estímulos auditivos y sus respectivas ubicaciones utilizando métodos tradicionales. Para ello se seleccionaron pruebas específicas, que fueron las siguientes: TOMAL [24], EDAF [25], y ECM [26]. Para la evaluación de la memoria visuoespacial se seleccionó el sub-test de la batería TOMAL: Memoria de Lugares (ML). En este sub-test, el participante tiene que identificar la ubicación de unos puntos en unas cuadrículas. El rango de las cuadrículas es de $3 \times 3$ y $4 \times 4$. Con el fin de evaluar el recuerdo inmediato de elementos auditivos, se utilizaron dos subpruebas de amplitud de memoria verbal de la batería TOMAL: Dígitos Directos (DD) y Dígitos Inversos (DI). DD es una tarea que mide el recuerdo de una secuencia de números en el mismo orden que el indicado por el evaluador. La tarea DI (una variación de la tarea DD) consiste en recordar una secuencia de números, pero en orden inverso. Para la evaluación de la discriminación auditiva y fonológica, se seleccionaron las subpruebas de EDAF: Memoria Secuencial Auditiva (MSA), y Discriminación de Sonidos del Medio (DSM). MSA es una tarea de repetición de palabras. DSM es una tarea de discriminación de sonidos (p. ej., el llanto del bebé, el ruido del tráfico, etc.). EDAF está compuesto de pruebas que evalúan la capacidad humana de procesamiento auditivo y, en caso de utilizarse para fines diagnósticos, resulta adecuado en población de entre 2 y 7 años. Sin embargo, EDAF se ha utilizado para la evaluación de posibles dificultades de discriminación auditiva después de los 7 años (p. ej., SantiagoPardo et al. (2018) lo utilizaron en personas de entre 3 y 15 años). Versiones similares de las pruebas utilizadas en EDAF también se han utilizado para obtener un índice de rendimiento en tareas de percepción y discriminación auditiva en diferentes rangos de edad (por ejemplo, Niejenhuis et al., 2002; Humes et al., 2003). Niejenhuis et al. (2002) estudiaron el rendimiento de grupos de niños y adultos de entre 8 y 57 años con una tarea similar. Por último, se utilizaron algunas preguntas de la Escala Clínica de Memoria (ECM) para evaluar la memoria espacial del participante en el día a día.

TABLA II

CUESTIONARIO DE USABILIDAD Y SATISFACCIÓN

\begin{tabular}{ll}
\hline \hline No. & \multicolumn{1}{c}{ Pregunta } \\
\hline PU1 & El juego fue fácil de usar \\
PU2 & Fue fácil aprender a usar este juego \\
PS1 & Lo he pasado bien \\
PS2 & Se lo recomendaría a un amigo \\
PS3 & En general, estoy satisfecho con este juego \\
PS4 & Me ha gustado moverme para localizar la fuente del \\
PS5 & Sonido he hastado jugar con los Karotz \\
\hline \hline
\end{tabular}

TABLA III

PRUEBA $U$ DE MANN-WHITNEY PARA LAS VARIABLES OBTENIDAS DURANTE EL JUEGO (GRUPOS A Y B)

\begin{tabular}{|c|c|c|c|c|c|c|}
\hline Var. & Grupo A & Grupo B & $\bar{U}$ & $\overline{Z Z}$ & 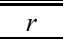 & $\overline{p p}$ \\
\hline NEA & $20.0 \pm 10.5$ & $39.0 \pm 6.9$ & 181.5 & -7.38 & 0.74 & $<0.001^{*}$ \\
\hline $\mathrm{NNC}$ & $1.4 \pm 0.7$ & $3.0 \pm 1.3$ & 389.5 & -6.16 & 0.62 & $<0.001^{*}$ \\
\hline TT & $10.0 \pm 3.7$ & $11.1 \pm 2.2$ & 991.0 & -1.79 & 0.18 & 0.074 \\
\hline
\end{tabular}

Se diseñó un cuestionario de usabilidad y satisfacción para evaluar la experiencia de los participantes y sus percepciones al utilizar el juego, Tabla II. Para su diseño, se utilizaron como base los cuestionarios de Lewis [30] y de Lund [31]. Los participantes respondieron a las preguntas utilizando una escala Likert de 5 puntos (de 1 = "totalmente en desacuerdo" a $5=$ "totalmente de acuerdo").

\section{RESUltados}

Los datos del estudio se analizaron utilizando $\mathrm{R}$, versión 3.2.1, y RStudio, versión 0.99 (https://www.r-project.org). En primer lugar, se realizó un análisis descriptivo inicial. A continuación, se comprobó la normalidad de los datos utilizando las pruebas Shapiro-Wilk y Anderson-Darling. Los datos no se ajustan a una distribución normal. Por lo que se han utilizado pruebas no paramétricas ( $U$ de Mann-Whitney y Kruskal-Wallis). En las tablas, el símbolo '*' indica diferencias significativas, y cuando aparecen dos valores separados por ' \pm ' indican medias y desviaciones estándar (por ejemplo, en las columnas Grupo A y Grupo B de la Tabla III).

\section{A. Resultados del juego}

La Tabla III muestra los resultados del juego para los Grupos A y B. Se observa que el rendimiento del Grupo A fue estadísticamente menor que el del Grupo B (NEA y NNC). No hubo diferencias significativas en el tiempo empleado entre ambos grupos (TT).

La Tabla IV muestra los resultados del juego para los Grupos B y C. Se observa que el rendimiento de los niños fue estadísticamente menor que el de los adultos (NEA y NNC). También, hubo diferencia estadísticamente significativa en el tiempo empleado entre ambos grupos (TT), los niños necesitaron un tiempo mayor.

Considerando los dos grupos de niños (Grupos A y B), se realizaron pruebas de Kruskal-Wallis para determinar la influencia de tres factores (Género, Edad, y Grupo) en la variable NNC, Tabla V. Los resultados muestran que hubo diferencias significativas para la Edad y el Grupo, pero no para el Género. Como se observa en la Tabla III, los niños de 8 a 10 años presentan un rendimiento significativamente mejor que los niños de 5 a 7 años.

\section{TABLA IV}

PRUEBA $U$ DE MANN-WHITNEY PARA LAS VARIABLES OBTENIDAS DURANTE EL JUEGO (GRUPOS B Y C)

\begin{tabular}{lrrrrrc}
\hline \hline Var. & Grupo B & Grupo C & $U$ & $Z$ & $r$ & $p$ \\
\hline NEA & $39.0 \pm 6.9$ & $43.8 \pm 1.8$ & 497.0 & -5.10 & 0.52 & $<0.001^{*}$ \\
NNC & $3.0 \pm 1.3$ & $4.3 \pm 1.0$ & 507.5 & -5.12 & 0.52 & $<0.001^{*}$ \\
TT & $11.1 \pm 2.2$ & $8.7 \pm 1.0$ & 2037 & 5.95 & 0.60 & $<0.001^{*}$ \\
\hline \hline
\end{tabular}

TABLA V

Prueba de KRUSKAL-WALlis PARA LA VARIABLE NNC, CONSIDERANDO LOS GRUPOS A Y B

\begin{tabular}{lccc}
\hline \hline \multicolumn{1}{c}{ Factores } & \multicolumn{1}{c}{$\chi^{2}$} & d.f. & $p$ \\
\hline Género & 0.001 & 1 & 0.983 \\
Edad & 49.299 & 5 & $<0.001^{*}$ \\
Grupo (A/B) & 37.944 & 1 & $<0.001^{*}$ \\
\hline \hline
\end{tabular}




\section{B. Resultados utilizando métodos tradicionales}

La Tabla VI muestra los resultados del análisis estadístico aplicado a los datos obtenidos por los Grupos A y B en los métodos tradicionales. Las puntuaciones en las subpruebas ML (Memoria de Lugares), DD (Dígitos Directos), y ECM (Evaluación Clínica de la Memoria- memoria espacial en el día a día) no ofrecen diferencias significativas entre los dos grupos respecto a su capacidad para recordar ubicaciones espaciales, secuencias de sonidos verbales, y su memoria espacial en el día a día. Hubo diferencias significativas entre los dos grupos en las valoraciones de las subpruebas DI (Dígitos Inversos) y DSM (Discriminación de Sonidos del Medio).

Se realizaron pruebas de Kruskal-Wallis para determinar la influencia de tres factores (Género, Edad, y Grupo) en la subprueba DSM, considerando los niños, Tabla VII. Los resultados muestran que hubo diferencias significativas para la Edad y el Grupo, pero no para el Género. Como se observa en la Tabla VI, los niños mayores obtienen un rendimiento significativamente mejor que los niños más pequeños en la discriminación de sonidos.

La Tabla VIII muestra los resultados del análisis estadístico aplicado a los datos obtenidos utilizando métodos tradicionales y para los Grupos B y C. Las puntuaciones en las subpruebas muestran diferencias significativas entre los dos grupos a favor de los adultos en todas las pruebas, a excepción de la prueba DD. Pero, en todas las pruebas los adultos obtuvieron una puntuación mayor que los niños.

\section{Correlaciones entre el juego y métodos tradicionales}

Para comparar el rendimiento de los participantes al utilizar el juego (NNC) y los métodos tradicionales (ML, DI, DSM y ECM), se han calculado las correlaciones con la muestra completa (100 niños y 48 adultos). Se aplicó la correlación de Spearman y se obtuvo el tamaño del efecto de dicha correlación, rho $(\rho)$. Las correlaciones entre la variable NNC y las variables de métodos tradicionales son las siguientes: ML $(\rho=0.49, p<$ $0.001 *)$, DI $(\rho=0.62, p<0.001 *)$, DSM $(\rho=0.34, p<0.001 *)$ y $\operatorname{ECM}\left(\rho=0.43, p<0.001^{*}\right)$. Todas las correlaciones son lineales y positivas. Estas correlaciones demuestran la similitud entre nuestro juego y los métodos tradicionales.

\section{Resultados de usabilidad y satisfacción}

Se aplicó la prueba $U$ de Mann-Whitney a los datos obtenidos del cuestionario de usabilidad y satisfacción. Los resultados para el Grupo A y B se muestran en la Tabla IX. Los resultados del Grupo B y C se muestran en la Tabla X. Existen diferencias significativas entre los grupos A y B, para las preguntas PS1 y PS5. Los niños mayores se han divertido más y les ha gustado más jugar con los Karotz. Sin embargo, ambos grupos otorgaron una puntuación muy alta a las preguntas de usabilidad y satisfacción (la media más baja fue de 4.28 en una escala de 1 a 5).

Al comparar el grupo de los niños mayores (Grupo B) con el grupo de los adultos (Grupo C), se observa que existen diferencias significativas en todas las preguntas, a favor de los niños, excepto en la PU2 (Fue fácil aprender a usar este juego).
TABLA VI

PRUEBA $U$ DE MANN-WhitNEY APLICAdA A Las PUNTUACIONES OBTENIDAS UTILIZANDO MÉTODOS TRADICIONALES Y CONSIDERANDO LOS GRUPOS A Y B

\begin{tabular}{lrrrccc}
\hline \hline Var. & Grupo A & Grupo B & \multicolumn{1}{c}{$U$} & \multicolumn{1}{c}{$\mathrm{Z}$} & $\mathrm{r}$ & $p$ \\
\hline ML & $10.6 \pm 6.2$ & $12.3 \pm 7.5$ & 1074.5 & -1.212 & 0.121 & 0.227 \\
DD & $8.1 \pm 1.8$ & $8.1 \pm 1.9$ & 1282.5 & 0.230 & 0.023 & 0.821 \\
DI & $13.2 \pm 6.5$ & $20.0 \pm 7.7$ & 426.5 & -5.688 & 0.569 & $<0.001^{*}$ \\
DSM & $13.6 \pm 1.1$ & $14.1 \pm 0.9$ & 934.5 & -2.279 & 0.228 & $0.023^{*}$ \\
ECM & $3.9 \pm 3.3$ & $3.4 \pm 2.8$ & 1341.0 & 0.633 & 0.063 & 0.530 \\
\hline \hline
\end{tabular}

TABLA VII

Prueba de Kruskal-Wallis para una de las Pruebas Tradicionales (DSM) EN LOS NIÑOS

\begin{tabular}{lrcl}
\hline \multicolumn{1}{c}{ Factores } & $\chi^{2}$ & d.f. & \multicolumn{1}{c}{$p$} \\
\hline Género & 1.120 & 1 & 0.290 \\
Edad & 17.223 & 5 & $0.004^{*}$ \\
Grupo (A/B) & 5.193 & 1 & $0.022^{*}$ \\
\hline \hline
\end{tabular}

TABLA VIII

PRUEBA $U$ DE MANN-WHITNEY APLICADA A LAS PUNTUACIONES OBTENIDAS UTILIZANDO MÉTODOS TRADICIONALES Y CONSIDERANDO LOS GRUPOS B Y C

\begin{tabular}{lrrrccc}
\hline Var. & \multicolumn{1}{c}{ Grupo B } & \multicolumn{1}{c}{ Grupo C } & \multicolumn{1}{c}{ U } & \multicolumn{1}{c}{ Z } & r & p \\
\hline ML & $12.3 \pm 7.5$ & $18.8 \pm 5.0$ & 546.5 & -4.65 & 0.47 & $<0.001^{*}$ \\
DD & $8.1 \pm 1.9$ & $8.7 \pm 2.6$ & 339.0 & -0.58 & 0.07 & 0.574 \\
DI & $20.4 \pm 7.6$ & $36.5 \pm 13.7$ & 303.5 & -6.38 & 0.64 & $<0.001^{*}$ \\
DSM & $14.1 \pm 0.9$ & $14.7 \pm 0.5$ & 762.5 & -3.46 & 0.35 & $<0.001^{*}$ \\
ECM & $3.4 \pm 2.8$ & $11.6 \pm 4.4$ & 114.0 & -7.74 & 0.78 & $<0.001^{*}$ \\
\hline
\end{tabular}

TABLA IX

PRUEBa $U$ de MANN-WhitNey PARA LAS PREgunTAS DE USABILIDAd Y SATISFACCIÓN, CONSIDERANDO LOS GRUPOS A Y B

\begin{tabular}{lcccccc}
\hline \hline Preg. & Grupo A & Grupo B & $U$ & $Z$ & $r$ & $p$ \\
\hline PU1 & $4.76 \pm 0.56$ & $4.80 \pm 0.40$ & 1245 & -0.050 & 0.005 & $<0.99$ \\
PU2 & $4.70 \pm 0.54$ & $4.78 \pm 0.42$ & 1189 & -0.567 & 0.057 & 0.577 \\
PS1 & $4.76 \pm 0.56$ & $4.96 \pm 0.20$ & 1072 & -2.261 & 0.226 & $0.030^{*}$ \\
PS2 & $4.64 \pm 0.83$ & $4.74 \pm 0.53$ & 1237 & -0.124 & 0.012 & 0.875 \\
PS3 & $4.64 \pm 0.63$ & $4.76 \pm 0.48$ & 1160 & -0.822 & 0.082 & 0.426 \\
PS4 & $4.38 \pm 0.99$ & $4.44 \pm 0.76$ & 1263 & 0.103 & 0.010 & 0.931 \\
PS5 & $4.28 \pm 0.78$ & $4.64 \pm 0.53$ & 953 & -2.317 & 0.232 & $0.024^{*}$ \\
\hline \hline
\end{tabular}

TABLA X

Prueba $U$ DE MANN-WhITNEY PARA LAS PREGUNTAS DE USABILIDAD Y SATISFACCIÓN, CONSIDERANDO LOS GRUPOS B Y C

\begin{tabular}{lcccccc}
\multicolumn{7}{c}{ SATISFACCION, CONSIDERANDO LOS GRUPOS B Y C } \\
\hline \hline Preg. & Grupo B & Grupo C & $U$ & $Z$ & $r$ & $p$ \\
\hline PU1 & $4.80 \pm 0.40$ & $4.23 \pm 0.66$ & 1780.0 & 4.743 & 0.479 & $<0.001^{*}$ \\
PU2 & $4.78 \pm 0.42$ & $4.83 \pm 0.38$ & 1136.0 & -0.66 & 0.067 & 0.612 \\
PS1 & $4.96 \pm 0.20$ & $4.25 \pm 0.56$ & 1980.0 & 6.628 & 0.669 & $<0.001^{*}$ \\
PS2 & $4.74 \pm 0.53$ & $4.04 \pm 0.82$ & 1804.5 & 4.804 & 0.485 & $<0.001^{*}$ \\
PS3 & $4.76 \pm 0.48$ & $4.38 \pm 0.61$ & 1614.0 & 3.446 & 0.348 & $<0.001^{*}$ \\
PS4 & $4.44 \pm 0.76$ & $3.85 \pm 0.90$ & 1627.0 & 3.262 & 0.330 & $0.001^{*}$ \\
PS5 & $4.64 \pm 0.53$ & $4.27 \pm 0.64$ & 1569.5 & 2.962 & 0.299 & $0.024^{*}$ \\
\hline \hline
\end{tabular}

VI. DISCUSIÓN

Nuestro juego implica la evaluación simultánea de habilidades espaciales y auditivas. Por contra, los métodos tradicionales consideran cada habilidad por separado. Estos métodos son pruebas específicas de tipo lápiz y papel, TOMAL [24], EDAF [25], y ECM [26]. Se han encontrado correlaciones entre nuestro juego y métodos tradicionales, indicando que nuestro juego ha demostrado ser una herramienta válida para evaluar la memoria espacial tanto para niños como para adultos. Hecho que corrobora nuestra hipótesis. Por lo que sabemos, ningún estudio ha probado este tipo de evaluación utilizando la 
tecnología propuesta. Respecto a los sistemas de realidad virtual [21] [22] o aumentada [18] [19] presentados hasta la fecha se han centrado en estímulos visuales. Sin embargo, nuestra propuesta comparte con trabajos previos que los estímulos estén ubicados en el entorno real y que el usuario tenga que caminar hasta llegar a dicha ubicación [18] [19]. Respecto a trabajos previos que hayan utilizados estímulos auditivos, Parmentier et al. [32] utilizaron altavoces ubicados en diferentes lugares de una habitación. El participante permanecía sentado en el centro de la misma y realizaba la tarea en la oscuridad. El participante debía señalar en una pantalla el altavoz que creía que había emitido el sonido. En otro trabajo, los altavoces emitían sonidos frente o alrededor de la cabeza de la persona que permanecía estática [33]. Las interfaces de usuario naturales aplicadas a esta necesidad suponen una ventaja respecto a los trabajos previos, ya que la información espacial de la fuente del sonido no se está considerando porque se anula el desplazamiento hasta el origen, actividad que ocurre en una situación natural.

El rendimiento con el juego ha sido significativamente incremental y directamente relacionado con el grupo de edad (Grupo A < Grupo B < Grupo C). El juego requiere atención y concentración constantes, dado que implica la evaluación simultánea de habilidades espaciales y auditivas. Que los resultados hayan sido mejores para los adultos y los niños mayores, por este orden, es consistente con la idea de que los adultos pueden almacenar más elementos en la memoria a corto plazo que los niños, debido a que la capacidad de memoria aumenta a lo largo del desarrollo evolutivo hasta su maduración en la etapa adulta [34].

Respecto a la usabilidad y satisfacción con el juego, entre los niños no ha habido diferencias significativas para las 8 preguntas, excepto para la PS1 (Lo he pasado bien) y PS5 (Me ha gustado jugar con los Karotz), a favor de los niños mayores. Sin embargo, ambos grupos otorgaron una puntuación muy alta a las preguntas de usabilidad y satisfacción (la media más baja fue de 4.28 en una escala de 1 a 5). Por lo que se puede concluir, que a todos los niños les ha parecido fácil de aprender a usar y fácil de utilizar, que se lo han pasado bien, y que han mostrado su satisfacción con el juego. Dado que el juego les ha parecido fácil de utilizar, los niños han podido concentrarse en las tareas a realizar, en lugar de la interacción en sí. Los Karotz emiten sonidos y mueven sus orejas e iluminan su parte central, por lo que los niños los pudieron considerar como mascotas o juguetes. El uso de múltiples modalidades sensoriales puede influir en la satisfacción de los niños. La comunicación de los participantes con los Karotz levantando los brazos se puede interpretar como un código de comunicación entre iguales, que puede influir en lo atractivo del juego. Muchos niños estaban acostumbrados a este tipo de interacción dada la proliferación de juegos que utilizan Kinect, y por ello este tipo de interacción les parecía natural. Desde nuestro punto de vista, todo ello contribuye a la inmersión que induce nuestro juego y a la motivación demostrada por parte de los participantes. Las observaciones de la supervisora corroboran nuestra percepción de inmersión. La supervisora añade que muchos niños no se daban cuenta de que ella estaba allí y que incluso, hablaban con los Karotz.
Considerando la comparación entre los niños mayores y los adultos, se encontraron diferencias significativas en todas las preguntas, a favor de los niños, excepto en la PU2 (Fue fácil aprender a usar este juego), que ambos grupos consideraron que fue fácil de aprender (medias de 4.78 (niños) y 4.83 (adultos)). Los niños mayores otorgaron una puntuación muy alta a las preguntas de usabilidad y satisfacción (la media más baja fue de 4.44 en una escala de 1 a 5). Los adultos otorgaron valores inferiores, estando la media alrededor de 4 en todas las preguntas, excepto en PU2 que fue de 4.83. Por lo que, se puede concluir que, el juego, a los adultos también les pareció fácil de aprender a usar y fácil de utilizar, que se lo pasaron bien, y mostraron su satisfacción con el mismo. Sin embargo, las diferencias significativas obtenidas en 6 de las 7 preguntas, a favor de los niños, indican que el juego sería especialmente atractivo para niños. Estas diferencias se podrían explicar considerando la brecha generacional entre niños y adultos, que implica disparidad en la capacidad y el conocimiento de la tecnología [35]. A este respecto, Prensky [36] argumenta que los niños son 'nativos digitales' y que los adultos son 'inmigrantes digitales'.

Con respecto a la interacción y a la selección de los Karotz, en este trabajo se ha utilizado la interacción mediante gestos. Sin embargo, se podría haber utilizado el botón que los Karotz tienen en la parte superior, utilizar una interfaz de usuario de lenguaje natural, seleccionar en una Tablet el Karotz correcto, etc. Como trabajo futuro se está estudiando la incorporación de otro tipo de interacción y su comparación. No obstante, nuestra propuesta pretendía que el juego pudiese ser utilizado por población invidente y la interacción alternativa también tendría que considerar este colectivo, por lo que, por ejemplo, pulsar el botón de la parte superior de los Karotz o utilizar una Tablet no serían alternativas viables. Respecto a los Karotz, para reproducir o mejorar nuestra propuesta se podría utilizar otro tipo de robots que actualmente estén en el mercado o que pudieran aparecer en el futuro.

Nuestro juego se podría comparar con otros métodos con el mismo principio, por ejemplo, utilizando como base la idea del juego "el director de orquesta". En este juego, un grupo de niños se coloca en círculo mirando todos hacia el interior del mismo, mientras un niño del grupo queda fuera (niño $A$ ). Se nombra a un director de orquesta, sin que el niño $A$ sepa quién es. El director de orquesta indica un sonido que los demás niños tienen que imitar. El objetivo del niño $A$ es adivinar quién es el director. A su vez, el director tiene que cambiar el sonido sin que el que busca al director se dé cuenta, y los demás le siguen. El juego termina cuando se adivina quién es el director de orquesta. Para la comparativa con nuestro juego, se podría utilizar una variante del juego "el director de orquesta", en el que el director de orquesta haría las tareas del ordenador de nuestro juego, el resto de niños harían las tareas de los Karotz, y el niño $A$ sería el participante en nuestro juego.

\section{CONCLUSIONES}

Nuestro juego permite evaluar la integración multimodal de la información auditiva y espacial tanto en niños como en adultos. El juego podría ser una buena herramienta para identificar alteraciones en la memoria espacial tanto en niños como en adultos. En los niños se podría realizar una 
identificación temprana del desarrollo atípico, niños con trastorno por déficit de atención e hiperactividad (TDAH), etc. En los adultos se podría utilizar para identificar alteraciones en su memoria, como por ejemplo, la enfermedad de Alzheimer, otros tipos de demencia con alteraciones de la orientación, pacientes con secuelas tras sufrir un accidente cerebrovascular o traumatismo craneal, etc.

En otro trabajo futuro, el juego se podría mejorar combinando claves visuales y auditivas (p. ej., se podrían incluir como claves visuales los movimientos de los robots). En este trabajo, hemos comparado niños y adultos, pero también son posibles otras comparaciones. Por ejemplo, sería interesante comparar el rendimiento de los niños con visión normal y los niños ciegos o usar este nuevo juego con niños con TDAH. El conocimiento sobre cómo aprenden en diferentes entornos inteligentes proporcionaría una base para entender sus fortalezas y debilidades cognitivas, además de contribuir al desarrollo de nuevas formas de aprendizaje.

\section{AgRAdECIMIENTOS}

Este trabajo ha sido financiado fundamentalmente por el Ministerio de Economía y Competitividad (MINECO) a través del proyecto CHILDMNEMOS (TIN2012-37381-C02-01) y cofinanciado con fondos FEDER (Fondo Europeo de Desarrollo Regional).

Otras ayudas financieras se han recibido del Gobierno de la República de Ecuador a través del Programa de Becas de la Secretaría de Educación Superior, Ciencia, Tecnología e Innovación (SENESCYT); del Gobierno de Aragón (Departamento de Industria e Innovación), y el Fondo Social Europeo para Aragón.

Nos gustaría agradecer sus contribuciones a: Jimena Bonilla; y a los usuarios que han participado en este estudio.

\section{REFERENCIAS}

[1] N. Burgess, G. Hitch, "Computational models of working memory: putting long-term memory into context," Trends Cogn Sci, vol. 9, pp. 535-541, 2005, 10.1016/j.tics.2005.09.011.

[2] N. Burgess, S. Becker, J. A. King, J. O'Keefe, "Memory for events and their spatial context: models and experiments," Philos Trans R Soc Lond B Biol Sci, vol. 356, pp. 1493-1503, 2001, 10.1098/rstb.2001.0948.

[3] B. P. Rourke, "Arithmetic disabilities, specific and otherwise: A neuropsychological perspective," J. Learn. Disabil, vol. 26, pp. 214-226, 1993, 10.1177/002221949302600402.

[4] M. J. Dehn, "Cognitive Processing Deficits," in Evidence-Based Interventions for Students with Learning and Behavioral Challenges, R.J. Morris, N. Mather, Ed., New York, London: Routledge, 2008, pp. 258287.

[5] F. H. Rauscher, R. M. Krauss, Y. Chen, "Gesture, Speech, and Lexical Access: The Role of Lexical Movements in Speech Production," Psychol. Sci, vol. 7, pp. 226-231, 1996, 10.1111/j.1467-9280.1996.tb00364.x.

[6] C. Cuerda, J. A. Gallud, "Forgettify: An Application to Help People with Memory Limitations," IEEE Latin America Transactions, vol. 16, no. 8 , pp. 2261-2266, 2018, 10.1109/TLA.2018.8528244.

[7] J. F. Martín-SanJosé, M. C. Juan, E. Torres, M. J. Vicent, "Playful interaction for learning collaboratively and individually," Journal of Ambient Intelligence and Smart Environments, vol. 6, no. 3, pp. 295-311, 2014, 10.3233/AIS-140257.

[8] J. S. da Cunha Neto, V. H. C. de Albuquerque, G. P. F. Silva, N. B. C. Olegario, D. R. Pereira, J. M. R. S. Tavares, "GoNet - A New Movement Dynamic Evaluation System in Real Time," IEEE Latin America Transactions, vol. 13, no. 12, pp. 3928-3933, 2015, 10.1109/TLA.2015.7404929.
[9] H. Cheng, L. Yang, Z. Liu, "Survey on 3D Hand Gesture Recognition," IEEE Transactions on Circuits and Systems for video technology, vol. 26, no. 9, pp. 1659-1673, 2016, 10.1109/TCSVT.2015.2469551.

[10]J. Jimenez, A. Martin, V. Uc, A. Espinosa, "Mexican Sign Language Alphanumerical Gestures Recognition using 3D Haar-like Features," IEEE Latin America Transactions, vol. 15, no. 10, pp. 2000-2005, 2017, 10.1109/TLA.2017.8071247.

[11] R. C. Carro, E. B. Huerta, R. M. Caporal, J. C. Hernández, F. R. Cruz, "Facial Expression Analysis with Kinect for the Diagnosis of Paralysis Using Nottingham System," IEEE Latin America Transactions, vol. 14, no. 7, pp. 3418-3426, 2016, 10.1109/TLA.2016.7587650.

[12] Iu. G. Kryvonos, Iu. V. Krak, O. V. Barmak, R. O. Bagriy, "New Tools of Alternative Communication for Persons with Verbal Communication Disorders," Cybernetics and Systems Analysis, vol. 52, no. 5, pp. 665-673, 2016, 10.1007/s10559-016-9869-3.

[13]N. A. Malik, S. Shamsuddin, H. Yussof, M. A. Miskam, A. C. Hamid, "Feasibility of using a humanoid robot to elicit communicational response in children with mild autism," IOP Conference Series: Materials Science and Engineering, vol. 53, paper: 012077, 2013, 10.1088/1757$899 \mathrm{x} / 53 / 1 / 012077$.

[14]G. Keren, M. Fridin, "Kindergarten Social Assistive Robot (KindSAR) for children's geometric thinking and metacognitive development in preschool education: A pilot study," Computers in Human Behavior, vol. 35, pp. 400412, 2014. 10.1016/j.chb.2014.03.009.

[15]Y. Moriguchi, T. Kanda, H. Ishiguro, Y. Shimada, S. Itakura, "Can young children learn words from a robot?," Interact. Stud, vol. 12, pp. 107-118, 2011, 10.1075/is.12.1.04mor.

[16]C. Enríquez, O. Aguilar, F. Domínguez, "Using robot to motivate computational thinking in high school students," IEEE Latin America Transactions, vol. 14, no. 11, pp. 4620-4625, 2016 , 10.1109/TLA.2016.7795838.

[17]M. M. A. de Graaf, S. B. Allouch, T. Klamer, "Sharing a life with Harvey: Exploring the acceptance of and relationship-building with a social robot," Computers in Human Behavior, vol. 43, pp. 1-14, 2015, 10.1016/j.chb.2014.10.030.

[18]M. C. Juan, M. Mendez-Lopez, E. Perez-Hernandez, S. Albiol-Perez, "Augmented reality for the assessment of children's spatial memory in seal settings," PLOS ONE, vol. 9, no. 12, paper: e113751, 2014, 10.1371/journal.pone. 0113751 .

[19]F. Munoz-Montoya, F., M. C. Juan, M. Mendez-Lopez, C. Fidalgo, "Augmented Reality Based on SLAM to Assess Spatial Short-Term Memory," IEEE Access, vol. 7, pp. 2453-2466, 2019, 10.1109/ACCESS.2018.2886627.

[20]F. Munoz-Montoya, C. Fidalgo, M. C. Juan, M. Mendez-Lopez, "Memory for Object Location in Augmented Reality: The Role of Gender and the Relationship Among Spatial and Anxiety Outcomes," Frontiers in Human Neuroscience, vol. 13, Article N. 113, 2019, 10.3389/fnhum.2019.00113.

[21]D. Rodríguez-Andrés, M. C. Juan, M. Méndez-López, E. Pérez-Hernández, J. Lluch, "MnemoCity Task: Assessment of Childrens Spatial Memory Using Stereoscopy and Virtual Environments," PLOS ONE, vol. 11, no. 8, paper: e0161858, 2016, 10.1371/journal.pone.0161858.

[22] S. Cárdenas-Delgado, M. Méndez-López, M. C. Juan, E. Pérez-Hernández, J. Lluch, R. Vivó, "Using a Virtual Maze Task to assess spatial short-term memory in adults," in Proc. International Conference on Computer Graphics Theory and Applications, Porto, Portugal, 2017, pp. 46-57.

[23]K. Kalm, M. H. Davis, D. Norris, "Neural mechanisms underlying the grouping effect in short-term memory," Human Brain Mapping, vol. 33, no. 7, pp. 1634-1647, 2012, 10.1002/hbm.21308.

[24]C. R. Reynolds, E. D. Bigler, TOMAL test of memory and learning. Examiner's manual, Austin, TX: Pro-Ed [TOMAL Test de memoria y aprendizaje. Manual de interpretación (E. Goikoetxea, \& Departamento I+D de TEA Ediciones, Adapters), (2001, Madrid, Spain: TEA Ediciones)], 2001.

[25]M. F. Brancal, F. Alcantud, A. M. Ferrer, M. E. Quiroga, EDAF: Evaluación de la discriminación auditiva y fonológica, 2nd ed. Madrid: TEA Ediciones, 2009.

[26]K. W. Kamphaus, E. Perez-Hernandez, F. Sanchez-Sanchez, F, Cuestionario de Evaluación Clínica de la Memoria, In press. Madrid: TEA Ediciones, 2019.

[27]R. B.Santiago-Pardo, J.I. Benito-Orejas, A. L. Sanchez-Rosso, M. I. RicoPaino, A. Herrero-Galiacho, L.I. de Castro "Selection of tests of language and critical analysis of its application in children with hearing impairment," Revista de Investigacion en Logopedia, vol. 8, no. 2, pp. 147-164, 2018, 
[28]K. Niejenhuis, A. Snik, G. Priester, S. van Kordenoordt, P. van den Brock, "Age effects and normative data on a Dutch test battery for auditory processing disorders," International Journal of Audiology, vol. 41, pp. 334-346, 2002, 10.3109/14992020209090408.

[29] L. E. Humes, D. L. Wilson, A. C. Humes, "Examination of differences between successful and unsuccessful elderly hearing aid candidates matched for age, hearing loss and gender," International Journal of Audiology, vol. 42, no. 7, pp. 732-441, 2003, 10.3109/14992020309080053.

[30]J. R. Lewis, "IBM computer usability satisfaction questionnaires: Psychometric evaluation and instructions for use," International Journal of Human-Computer Interaction, vol. 7, no. 1, pp. 57-78, 1995, 10.1080/10447319509526110.

[31]A. M. Lund, Measuring Usability with the USE Questionnaire," STC Usability SIG Newsletter, vol. 8, no. 2, pp. 1-4, 2001.

[32]F. B. R. Parmentier, S. King, I. Dennis, "Local Temporal Distinctiveness does not Benefit Auditory Verbal and Spatial Serial Recall," Psychonomic Bulletin \& Review, vol. 13, no. 3, pp. 458-465, 2006 10.3758/BF03193870.

[33]G. H. Recanzone, "Where was that? - Human Auditory Spatial Processing," Trends in Cognitive Sciences, vol. 6, no. 8, pp. 319-320, 2002, 10.1016/S1364-6613(02)01951-4.

[34]D. L. T. Rohde, D. C. Plaut, "Less is less in language acquisition," in Connectionist Modeling of Cognitive Development, P. Quinlan, Ed., Hove, UK: Psychology Press, 2003, pp. 189-231.

[35] M. A. Warschauer, "A teacher's Place in the Digital Divide," Information and Communication Technologies, vol. 106, no. 2, pp. 147-166, 2007 10.1111/j.1744-7984.2007.00118.x.

[36] M. Prensky, "Listen to the Natives, "Educational Leadership, vol. 63, no. 4, pp. 8-13, 2005.

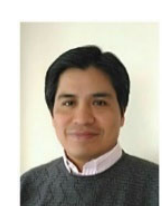

Mauricio Loachamín Valencia es Ingeniero de Sistemas e Informática (1996) y Máster en Gerencia de Sistemas (2005) por la Escuela Politécnica del Ejército (ESPE), Quito, Ecuador. Máster en Inteligencia Artificial, Reconocimiento de Formas e Imagen Digital (2013) por la Universitat Politècnica de València (UPV), y actualmente estudiante del Doctorado en Informática en la UPV. Desde 1996 es profesor en la ESPE y en la Universidad de las Américas.

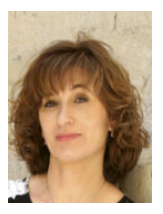

M. Carmen Juan es Licenciada en Informática (1994) y Doctora en Informática (2000) por la Universitat Politècnica de València (UPV). Desde 1996 es profesora en la UPV, y Catedrática de Universidad desde 2014. Sus áreas de interés incluyen: informática gráfica, realidad virtual y aumentada, interfaces de usuario avanzadas y sus aplicaciones a psicología, medicina y educación-entretenimiento.

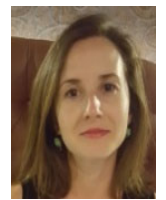

Magdalena Méndez López es Licenciada en Psicología (2004) y Doctora en Psicología (2009) por la Universidad de Oviedo. Desde 2010 es profesora de la Universidad de Zaragoza. Sus áreas de interés incluyen: orientación espacial, interfaces de usuario avanzadas, realidad virtual y aumentada, y sus aplicaciones a psicología.

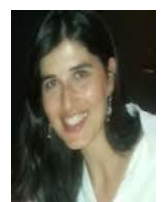

Elena Pérez Hernández es Licenciada en Psicología (2002) y Doctora en Psicología (2008) por la Universidad Complutense de Madrid. Es profesora de la Universidad Autónoma de Madrid. Sus objetivos de investigación se centran en: atención, memoria, bilingüismo y tecnologías aplicadas al estudio del desarrollo humano.

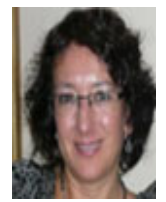

María José Vicent es Licenciada en Informática (1987) y Doctora en Informática (2003) por la Universitat Politècnica de València (UPV). Desde 1995 es profesora de la UPV y Profesora Titular de Universidad desde 2010. Sus áreas de interés incluyen: informática gráfica, síntesis de imagen, realidad virtual y aumentada e interfaces de usuario. 